\title{
Non-invasive Estimation of Pressure Changes along a Streamline using Vector Velocity Ultrasound
}

Olesen, Jacob Bjerring; Villagómez Hoyos, Carlos Armando; Traberg, Marie Sand; Jensen, Jørgen Arendt

\section{Published in:}

Proceedings of 2015 IEEE International Ultrasonics Symposium.

Link to article, DOI:

10.1109/ULTSYM.2015.0067

Publication date:

2015

Document Version

Peer reviewed version

Link back to DTU Orbit

Citation (APA):

Olesen, J. B., Villagómez Hoyos, C. A., Traberg, M. S., \& Jensen, J. A. (2015). Non-invasive Estimation of Pressure Changes along a Streamline using Vector Velocity Ultrasound. In Proceedings of 2015 IEEE International UItrasonics Symposium. IEEE. https://doi.org/10.1109/ULTSYM.2015.0067

\section{General rights}

Copyright and moral rights for the publications made accessible in the public portal are retained by the authors and/or other copyright owners and it is a condition of accessing publications that users recognise and abide by the legal requirements associated with these rights.

- Users may download and print one copy of any publication from the public portal for the purpose of private study or research.

- You may not further distribute the material or use it for any profit-making activity or commercial gain

- You may freely distribute the URL identifying the publication in the public portal 


\title{
Non-invasive Estimation of Pressure Changes along a Streamline using Vector Velocity Ultrasound
}

\author{
Jacob Bjerring Olesen, Carlos Armando Villagómez-Hoyos, Marie Sand Traberg, and Jørgen Arendt Jensen \\ Center for Fast Ultrasound Imaging, Dept. of Elec. Eng., Bldg. 349, \\ Technical University of Denmark, DK-2800 Kgs. Lyngby, Denmark
}

\begin{abstract}
A non-invasive method for estimating pressure changes along a streamline using ultrasound is presented. The suggested method estimates pressure gradients from 2-D vector velocity fields. Changes in pressure are derived using a model based on the Navier-Stokes equations. Scans of a carotid bifurcation phantom with a $70 \%$ constriction are performed using a linear array transducer connected to the experimental scanner, SARUS. 2-D fields of angle-independent vector velocities are acquired to a depth of $3 \mathrm{~cm}$ using directional synthetic aperture vector flow imaging. The performance of the suggested estimator is evaluated by comparing its results to a 3-D numerical simulation model. The study showed pressure drops across the constricted phantom varying from -5 Pa to $7 \mathrm{~Pa}$ with a standard deviation of $4 \%$. The proposed method had a normalised rootmean-square error of $10 \%$ in reference to the simulation model. Further, an in-vivo scan of the carotid bifurcation is made to show the feasibility of the technique in a less experimental environment.
\end{abstract}

\section{INTRODUCTION}

Abnormal changes in intravascular blood pressure are usually an indication of a diseased vessel. Measuring pressure variations is therefore used clinically as a diagnostic marker in assessing the physiological state of a cardiovascular region [1]. Intravascular pressure is currently assessed by inserting pressure sensing wires or catheters to the femoral artery and threading them to the region of interest. These procedures, however, suffer some severe limitation as they are highly invasive and require the use of ionizing radiation for guidance of the pressure sensory device. A recent report from De Vecchi et al. [2] demonstrated that the accuracy of using catheters is greatly dependent on the physical size and shape of the catheter. It was found that a $24 \%$ overestimation of the peak systolic pressure can result from using a routinely employed catheter compared to the gold standard using a wire.

In 1976, Holen et al. [3] introduced the first fully noninvasive alternative for estimating intravascular pressure based on Doppler ultrasound. Analysing audio signals of the frequency shifts received from the mitral jet revealed the peak systolic velocity. From this the local pressure gradients were calculated using an orifice equation. The method was attractive due to its avoidance of catheterization, but suffered from the drawback that it was solely reliant on a single velocity estimate, which made the method highly sensitive to hemodynamic factors unrelated to the constricted vessel's effect on the peak velocity, e.g. abnormal cardiac output. The method presented by Holen et al. was further studied in 1989 by Evans et al. [4], and later tested against clinical pressure catheters by Strauss- and Baumgartner et al. [5]-[7]. The latter studies agreed that non-invasive pressure estimation through the simplified Bernoulli equation was achievable, however, the obtained pressure estimates were greatly dependent on the size of the examined vessel and the examiners ability to correct for the Doppler angle. Further advancement in non-invasive techniques for improving pressure estimates have been proposed over the past decades [8]-[17], but none of these have as yet successfully managed to supercede pressure catheters in the clinic.

The purpose of this paper is to present a technique for estimating pressure changes from vector velocity ultrasound data, and to compare these to results obtained using a 3-D finite-element (FE) simulation model. The presented data are obtained from a flow model, which mimics a constricted carotid artery. The study concludes by presenting an in-vivo example of the proposed technique.

\section{Pressure Estimation using Vector Velocities}

The following section describes the employed method for calculating the pressure gradients. It is based on the NavierStokes equations:

$$
\rho\left[\frac{\partial \vec{v}}{\partial t}+\vec{v} \cdot \nabla \vec{v}\right]=-\nabla p+\rho \vec{g}+\mu \nabla^{2} \vec{v}
$$

presuming the conservation of mass and linear momentum. Eq. (1) describes the development of a fluid's velocity field $\vec{v}(\vec{r}, t)=\left(v_{x}(t), v_{y}(t), v_{z}(t)\right)$ by relating the forces acting on an incompressible volume to its acceleration and density throughout time, $t$, and space, $\vec{r}$. The left-hand side sums the local $\frac{\partial \vec{v}}{\partial t}$ and convective fluid acceleration $\vec{v} \cdot \nabla \vec{v}$, where $\rho$ is the density of the fluid and $\nabla$ is the spatial differential operator $\left(\frac{\partial}{\partial x}, \frac{\partial}{\partial y}, \frac{\partial}{\partial z}\right)$. The right-hand side shows the surface and volume forces that are responsible for the acceleration of the fluid. The forces constitute a pressure drop $-\nabla p$, a gravitational force $\vec{g}$, and a viscous drag caused by the viscosity of the fluid $\mu \nabla^{2} \vec{v}$, where $\nabla^{2} \vec{v}$ is the Laplacian of the velocity field. The gravitational term is usually neglected, as a patient undergoing an ultrasound scan is placed in a supine position, hence, the buoyancy force cancels out the gravitational force. Rewriting (1) into a scalar equation following a streamline, and where the influence of gravity is omitted, yields the following;

$$
\frac{\partial p}{\partial s}=-\rho\left[\frac{\partial v_{s}}{\partial t}+v_{s} \frac{\partial v_{s}}{\partial s}\right]+\mu \frac{\partial^{2} v_{s}}{\partial s^{2}}
$$


where $v_{s}$ is the scalar product of $\vec{v}(\vec{r}, t)$ and the vector that lies tangent to the streamline $d \vec{s}=\left(\hat{s}_{x}, \hat{s}_{y}, \hat{s}_{z}\right)$. Here $d \vec{s}$ is an element of distance along the streamline, which runs in the direction, $s$ Integrating (2) along the flow direction of the streamline gives the drop in pressure across the line,

$$
\Delta P(t)=\int_{0}^{L} \frac{\partial p}{\partial s} d s
$$

Eqs. (1)-(3) state that the three spatial vector components of $\vec{v}$ must be known to estimate the pressure gradient $\nabla p$. This study employs a velocity estimator, which yields the twodimensional (2-D) in-plane vector velocity field $\vec{v}=\left(v_{x}, v_{z}\right)$. The proposed method is, thus, developed assuming that the out-of-plane velocity $v_{y}$ is zero.

\section{A. Calculating the Temporal Acceleration}

The temporal derivative in (2) is approximated analytically by decomposing the measured flow profile into a series of sinusoids through a Fourier transform. The profile and its derivative are then expressed by a sum of sinusoids, which oscillate at the frequencies containing the highest levels of energies. Reconstructing the flow profile from a sum of sinusoids is possible as the flow is periodic over the cardiac cycle [4], [18]. The first order derivative of a given flow profile is expressed as:

$$
\frac{\mathrm{d} v_{s}(n, t)}{\mathrm{d} t} \approx-\sum_{p=1}^{N}\left|V_{p}(n)\right| 2 \pi f_{p} \sin \left(2 \pi f_{p} t+\varphi_{p}(n)\right),
$$

where $N$ is the number of sinusoids used in reconstructing the flow profile. $V_{p}$ and $\varphi_{p}$ are the amplitude and the phase of the frequency component $f_{p} . n$ is the index number that runs along the streamline. The selected frequencies, $f_{p}$, are chosen based on they level of energy in the frequency domain. For instance, blood flow in larger vessels is mainly govern by the pulsating motion of the heart, hence, the frequencies of highest energy levels are generally associated to the fundamental period of the heart cycles, and its harmonics.

\section{B. Calculating the Spatial Acceleration}

The spatial derivatives in (2) are, unlike the previous case, not necessarily periodic across the examined region. Thus, it is not possible to express the spatial acceleration by a sum of sinusoids. The derivatives are therefore calculated using polynomial filtering of the measured velocity field. A second-order polynomial is fitted to a subset of adjacent data points by the linear least-squared method. Convolution coefficients are calculated from the least-squared model, which are used for finding the first-order derivatives [19]. The spatial accelerations, $\frac{\partial}{\partial s}$ and $\frac{\partial^{2}}{\partial s^{2}}$, in (2) are calculated by pair-wise multiplication of the elements in the velocity window and the convolution coefficients $\left(B_{k}\right)$ before summing and scaling the five multiplication terms,

$$
\frac{\mathrm{d}^{k} v_{s}(n, t)}{\mathrm{d} s^{k}} \approx \frac{1}{\Delta s^{k}} \sum_{p=n-h_{w}}^{n+h_{w}} v_{s}(p, t) B_{k}\left(p-\left(n-h_{w}\right)+1\right) .
$$

Here $\Delta s$ is the sampling interval along the direction of the streamline. The index number $p$, is found from half the window size of the selected subset, calculated as: $h_{w}=\frac{N_{\text {set }}+1}{2}-1$, where $N_{s e t}$ is the number of samples in the subset. The convolution coefficients at $k$ equal to 1 and 2 , are $\vec{B}_{1}=\frac{1}{35}[-7,-3.5,0,3.5,7]$ and $\vec{B}_{2}=\frac{1}{35}[10,-5,-10,-5,10]$, respectively [19].

\section{Simulation Model}

The accuracy of the estimated pressure changes is evaluated through comparison to a FE model constructed in Comsol (Comsol v4.4, Comsol AB, Stockholm, Sweden). The geometry of the model is build from segmented MRI data of the flow phantom obtained using a 3-T scanner (Magnetom Trio, Siemens AG, Erlangen, Germany) at the Department of Diagnostic Radiology at Rigshospitalet, Denmark. The flow parameters of the simulation model are set to mimic the actual flow conditions in the experimental set-up.

\section{EXPERIMENTAL SET-UP AND EQUIPMENT}

Vector velocity data are acquired from the upper branch of a flow phantom mimicking the carotid bifurcation, (C70-SSEA Shelley Medical Imaging Technologies, Toronto, Canada). Measurements are made using a BK8670 linear array transducer connected to the experimental research scanner SARUS [20]. A three-cycle pulse with a center frequency of $7 \mathrm{MHz}$ is emitted at $12 \mathrm{kHz}$ to a depth of $3 \mathrm{~cm}$. Eight low-resolution images are summed for each high resolution image producing an effective frame-rate of $1,500 \mathrm{~Hz}$.

\section{Results}

Angle-independent velocities are estimated using directional synthetic aperture flow imaging, an approach explained by Villagomez-Hoyos et al. [21]. Flow data are recorded over three cardiac cycles, producing roughly 3,900 velocity frames.

The total pressure drop that exist across the scanned region is estimated as a function of time using (2). The spatial derivatives, $\frac{\partial v_{s}}{\partial s}$, which goes into the estimator, are calculated using polynomial filtering. A second-order polynomial is fitted to a subset of 71 adjacent velocity estimates covering a $1.4 \mathrm{~mm}$ line of the $10.7 \mathrm{~mm}$ long streamline. The window size and the order of the filter is selected to minimize the effect of estimator noise under the assumption that flow within a $1.4 \mathrm{~mm}$ region can be approximated by a second-order polynomial. Each individual gradient, $\frac{\partial p}{\partial s}$, gives an indication of how pressure at that particular position changes relative to neighbouring pressure values. Summing the discrete contributions from each estimate along the line, the relative drop in pressure that exist between the two ends of the streamline is obtained. The temporal evolution of the pressure drop for the three measured cardiac cycles is plotted in Fig. 1. The average standard deviation across the pulse is found to $4 \%$ in reference to the maximum pressure of $7 \mathrm{~Pa}$. A plot of the three pulses shown on top of each other is seen in Fig. 2. The mean of the three measured pressure profiles is plotted together with the simulated pressure drop from the FE model in Fig. 3. A normalized root-mean-square error of $10 \%$ is found between the estimated data and the reference model. 


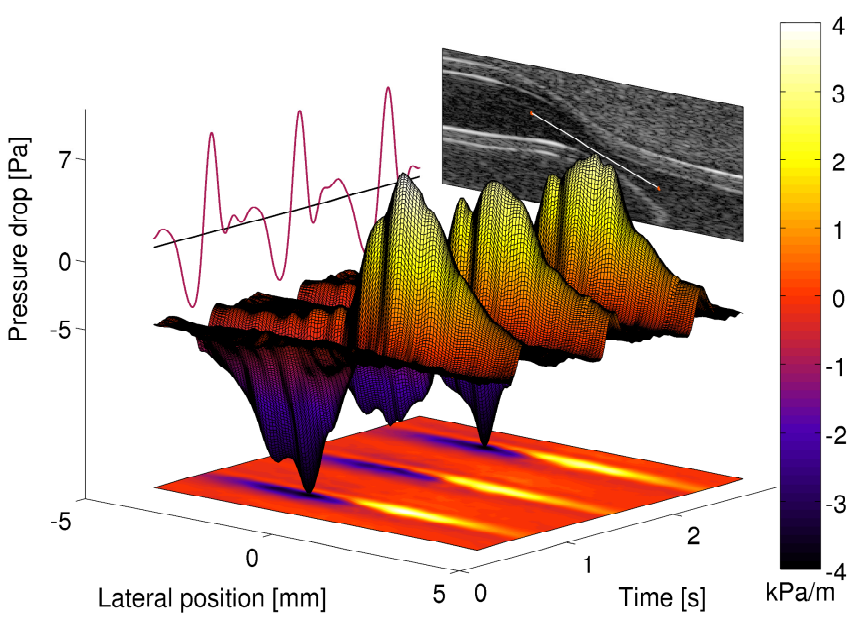

Fig. 1. Measured pressure drop across of the streamline seen on the B-mode image in the back. The center of the plot shows the individual pressure gradients along the line as a function of time. Summing the individual gradients along the streamline gives the total drop in pressure that exist across the stenotic region. This drop is presented by the red curve on the left.

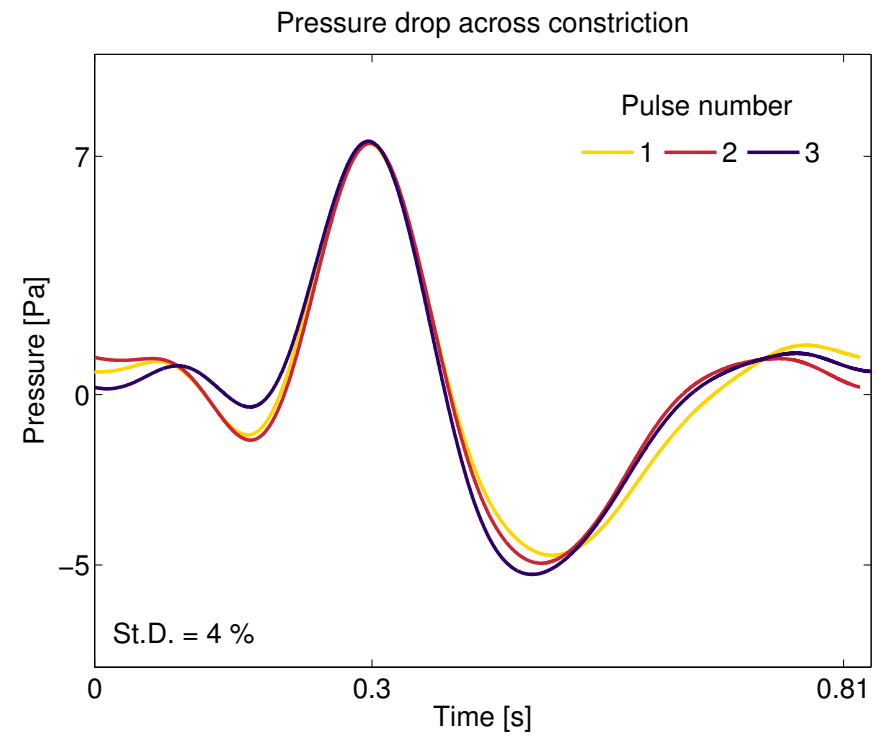

Fig. 2. Pressure drop between the two ends of the streamline for the three cardiac cycles.

\section{A. In-vivo Example}

An in-vivo measurement is carried out on the carotid artery of a healthy volunteer. The resulting vector flow image is shown in Fig. 4. The figure displays a longitudinal scan of the volunteer's left carotid bifurcations together with the measured flow field. The image is captured at peak systole, yielding values in the carotid sinus of roughly $0.6 \mathrm{~m} / \mathrm{s}$. Velocities along the streamline are extracted for all time instances and put into the proposed algorithm. The results are shown in Fig. 5. Relative pressure changes along the streamline are plotted as a function of time and lateral position. Here, the plot's left wall

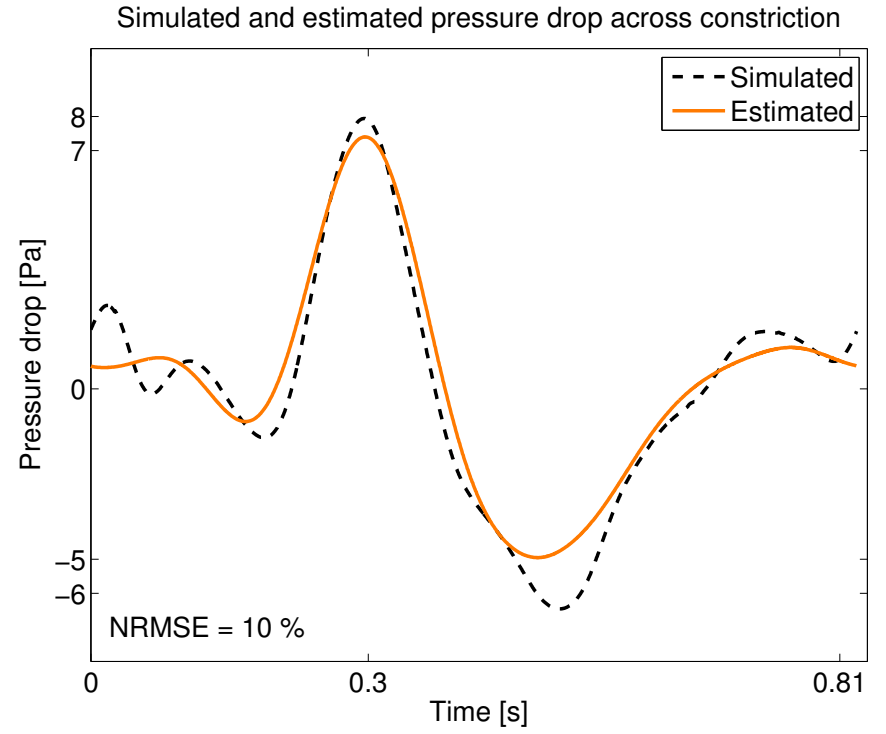

Fig. 3. Comparison of measured and simulated pressure drop. Dottedline: Simulated pressure drop across the constricted flow phantom. Solid-line: Mean estimated pressure drop from the three cardiac cycles measured using ultrasound.

makes up the starting point of the streamline data. Changes in pressure are then found relatively to this point, by moving down-right, parallel to the lateral axis. The figure shows an example on how intravascular pressure changes can be mapped from 2-D velocity data obtained using a non-invasive ultrasound technique.

\section{Discussion}

Non-invasive measurement of pressure changes have been calculated from vector velocity data. The pressure drop along a streamline varied depending on when in the cardiac cycle it was measured. Phantom measurements showed a standard deviation of $4 \%$, and a normalized error of $10 \%$. No previous studies on the topic have measured changes in pressure along streamlines using high frame-rate ultrasonic techniques. Such techniques otherwise allow for averaging across estimates without compromising the peak of the profile. Averaging is beneficial as it essentially performs a low-pass filtering of the estimates, thus, avoiding the higher frequency content, which usually is associated with noise. Noise cancellation is crucial for deriving proper derivatives, and becomes increasingly more important when moving into higher order derivatives.

Having a method that can detect changes in pressure directly from ultrasound flow data, will provide the clinician with a noninvasive tool for assessing the severity of a stenosis, without the need for ionizing radiation. This allows for the opportunity of making follow-up studies over the course of a disease without the discomfort associated with invasive procedures.

\section{ACKNOWLEDGMENTS}

The presented work has been financially supported by grant 024-2008-3 from the Danish Advanced Technology Foundation, BK Medical Aps, Denmark and Radiometer A/S, Denmark. 


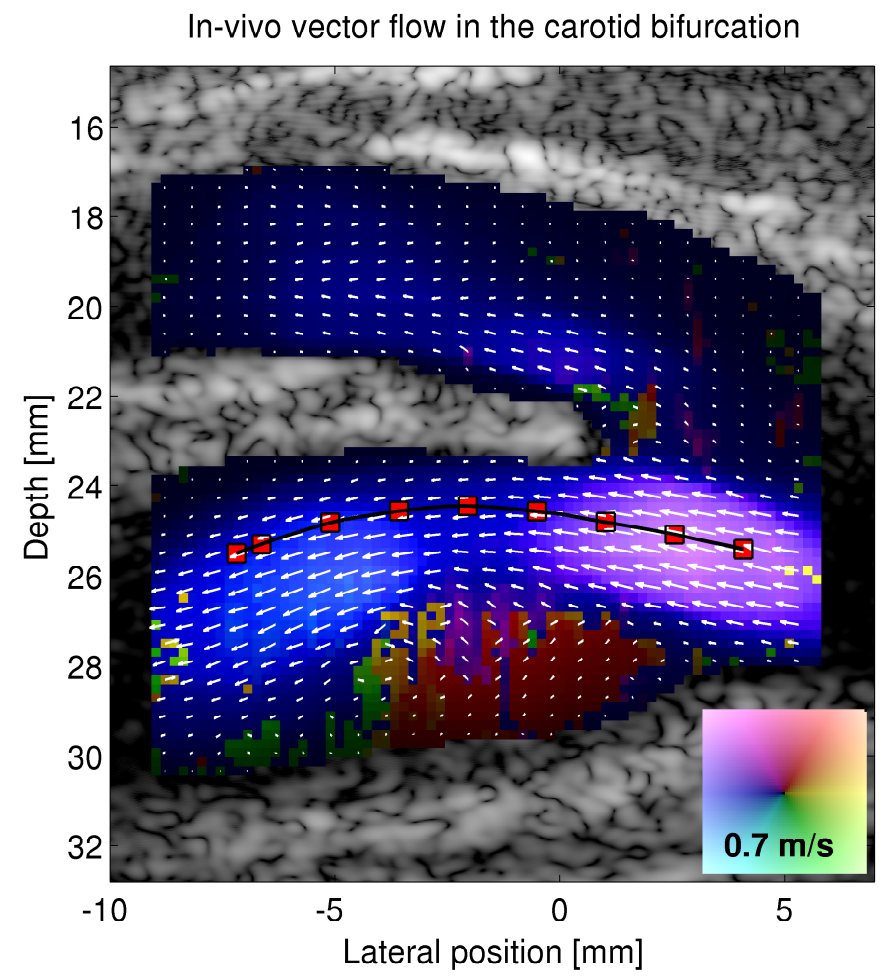

Fig. 4. Volunteer 1: Longitudinal vector flow scan of the carotid bifurcation during peak systolic. Bottom branch shows the internal carotid artery in conjunction with the carotid bulb, for which a vortex is formed during rapid flow movement. A streamline following the vector velocity field is also displayed.

\section{REFERENCES}

[1] D. S. Baim and W. Grossman, Grossman's cardiac catheterization angiography, and intervention. Lippincott Williams \& Wilkins, 2000.

[2] A. de Vecchi, R. E. Clough, N. R. Gaddum, M. C. M. Rutten, P. Lamata, T. Schaeffter, D. A. Nordsletten, and N. P. Smith, "Catheter-induced errors in pressure measurements in vessels: An in-vitro and numerical study," IEEE Trans. Biomed. Eng., vol. 61, no. 6, pp. 1844-1850, 2014.

[3] J. Holen, R. Aaslid, and K. Landmark, "Determination of pressure gradient in mitral stenosis with a non-invasive ultrasound Doppler technique," Acta med. scand., vol. 32, pp. 455-460, 1976.

[4] D. H. Evans, W. N. McDicken, R. Skidmore, and J. P. Woodcock, Doppler Ultrasound, Physics, Instrumentation, and Clinical Applications. New York: John Wiley \& Sons, 1989.

[5] A. L. Strauss, F. J. Roth, and H. Rieger, "Noninvasive assessment of pressure gradients across iliac artery stenoses: duplex and catheter correlative study," J. Ultrasound Med., vol. 12, no. 1, pp. 17-22, 1993.

[6] H. Baumgartner, H. Schima, G. Tulzer, and P. Kühn, "Effect of stenosis geometry on the Doppler-catheter gradient relation in vitro: A manifestation of pressure recovery," J. Am. Coll. Cardiol., vol. 21 , no. 4, pp. 1018-1025, 1993.

[7] H. Baumgartner, T. Stefenelli, J. Niederberger, H. Schima, and G. Maurer, "Overestimation of catheter gradients by Doppler ultrasound in patients with aortic stenosis: A predictable manifestation of pressure recovery," $J$ Am. Coll. Cardiol., vol. 33, no. 6, pp. 1655-1661, 1999.

[8] N. L. Greenberg, P. M. Vandervoort, M. S. Firstenberg, M. J. Garcia, and J. D. Thomas, "Estimation of diastolic intraventricular pressure gradients by Doppler M-mode echocardiography," Am. J. Physiol. Heart Circ. Physiol., vol. 280, no. 6, pp. 2507-2515, 2001.

[9] J. Bermejo, J. C. Antoranz, I. G. Burwash, J. L. Alvarez, M. Moreno, M. A. Garcia-Fernandez, and C. M. Otto, "In-vivo analysis of the instantaneous transvalvular pressure difference-flow relationship in aortic valve stenosis: implications of unsteady fluid-dynamics for the clinical

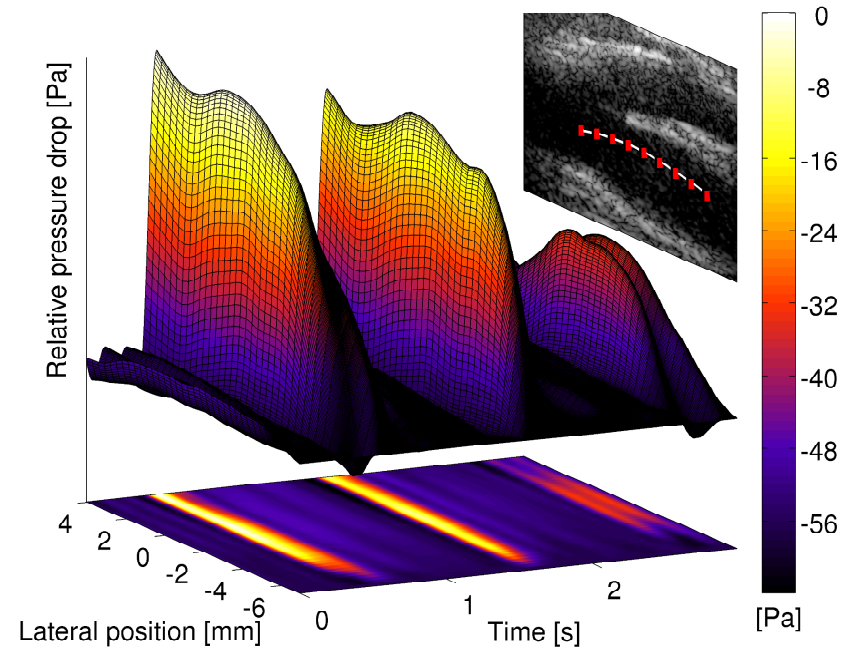

Fig. 5. In-Vivo: Changes in intravascular pressure measured as a function of time and lateral position along the streamline from Fig. 4. The shown pressures are in reference to an arbitrary value, which for this study is set to zero at the beginning of the streamline.

assessment of disease severity," J. Heart Valve Dis., vol. 11, no. 4, pp. $557-566,2002$.

[10] A. K. Reddy, G. E. Taffet, S. Madala, L. H. Michael, M. L. Entman, and C. J. Hartley, "Noninvasive blood pressure measurement in mice using Doppler ultrasound," Ultrasound Med. Biol., vol. 29, no. 3, pp. 379-385, 2003.

[11] S. Ohtsuki and M. Tanaka, "Doppler pressure field deduced from the Doppler velocity field in an observation plane in a fluid," Ultrasound Med. Biol., vol. 29, no. 10, pp. 1431-1438, 2003.

[12] J. M. Meinders and A. P. G. Hoeks, "Simultaneous assessment of diameter and pressure waveforms in the carotid artery," Ultrasound Med. Biol., vol. 30, no. 2, pp. 147-154, 2004.

[13] R. Yotti, J. Bermejo, J. C. Antoranz, J. L. Rojo-Alvarez, C. Allue, J. Silva, M. M. Desco, M. Moreno, and M. A. Garcia-Fernandez, "Noninvasive assessment of ejection intraventricular pressure gradients," J. Am. Coll. Cardiol., vol. 43, no. 9, pp. 1654-1662, 2004.

[14] J. D. Thomas and Z. B. Popovic, "Intraventricular pressure differences a new window into cardiac function," Circulation, vol. 112, pp. 1684-1686, 2005.

[15] D. Garcia, P. Pibarot, and L.-G. Durand, "Analytical modeling of the instantaneous pressure gradient across the aortic valve," J. Biomech., vol. 38, pp. 1303-1311, 2005.

[16] B. W. A. M. M. Beulen, N. Bijnens, G. G. Koutsouridis, P. J. Brands, M. C. M. Rutten, and F. N. van de Vosse, "Toward noninvasive blood pressure assessment in arteries by using ultrasound," Ultrasound Med. Biol., vol. 37, no. 5, pp. 788-797, 2011.

[17] J. B. Olesen, M. S. Traberg, M. J. Pihl, and J. A. Jensen, "Noninvasive esimation of 2-D pressure gradients in steady flow using ultrasound," IEEE Trans. Ultrason., Ferroelec., Freq. Contr., vol. 61, no. 8, pp. 14091418, 2014.

[18] J. R. Womersley, "Oscillatory motion of a viscous liquid in a thin-walled elastic tube. I: The linear approximation for long waves," Phil. Mag., vol. 46, pp. 199-221, 1955.

[19] A. Savitzky and M. J. E. Golay, "Smoothing and differentiation of data by simplified least squares procedures," Anal. Chem., vol. 36, no. 8, pp. $1627-1639,1964$.

[20] J. A. Jensen, H. Holten-Lund, R. T. Nilsson, M. Hansen, U. D. Larsen, R. P. Domsten, B. G. Tomov, M. B. Stuart, S. I. Nikolov, M. J. Pihl, Y. Du, J. H. Rasmussen, and M. F. Rasmussen, "SARUS: A synthetic aperture real-time ultrasound system," IEEE Trans. Ultrason., Ferroelec., Freq. Contr., vol. 60, no. 9, pp. 1838-1852, 2013.

[21] C. A. Villagomez-Hoyos, M. B. Stuart, and J. A. Jensen, "Adaptive multi-lag for synthetic aperture vector flow imaging," in Proc. IEEE Ultrason. Symp., 2014, pp. 1722-1725. 